\title{
Intravenous immunoglobulin in the treatment of primary trigeminal neuralgia refractory to carbamazepine: a study protocol[ISRCTN33042 I38]
}

\author{
Andreas Goebel ${ }^{* 1,2}$, Andrew Moore1, Rosamund Weatherall ${ }^{3}$, \\ Norbert Roewer ${ }^{2}$, Robert Schedel ${ }^{2}$ and Guenter Sprotte ${ }^{2}$
}

\begin{abstract}
Address: ${ }^{1}$ Nuffield Department of Anaesthetics, University of Oxford, Oxford Ox3 9DU, England, UK, ${ }^{2}$ Klinik für Anaesthesiologie, Julius Maximillians Universität Würzburg, 97080 Würzburg, Germany and ${ }^{3}$ Center for Statistics in Medicine, University of Oxford, Oxford OX3 7LF, England, UK

Email: Andreas Goebel* - andreasgoebel@rocketmail.com; Andrew Moore - andrew.moore@ pain-relief-unit.oxford.ac.uk; Rosamund Weatherall - R.Weatherall@icrf.icnet.uk; Norbert Roewer - nroewer@anaesthesie.uni-wuerzburg.de;

Robert Schedel - rschedel@anaesthesie.uni-wuerzburg.de; Guenter Sprotte -gsprotte@anaesthesie.uni-wuerzburg.de

* Corresponding author
\end{abstract}

This article is available from: http://www.biomedcentral.com/I47I-2377/3/I

(C) 2003 Goebel et al; licensee BioMed Central Ltd. This is an Open Access article: verbatim copying and redistribution of this article are permitted in all media for any purpose, provided this notice is preserved along with the article's original URL.

\begin{abstract}
Background: We have recently reported successful treatment of patients with chronic pain syndromes using human pooled intravenous immunoglobulin (IVIG) in a prospective, open-label cohort study. A randomised, placebo controlled, double blinded study is needed to confirm these results. We chose to study patients with carbamazepine resistant primary Trigeminal Neuralgia (rpTN), as these had responded particularly well to IVIG.

A protocol involving the use of IVIG in rPTN is complex for three reasons: I. The effect of IVIG does not follow simple dose-response rules; 2 . The response pattern of patients to IVIG was variable and ranged between no effect at all and pain free remission between two weeks and $>1$ year; 3. TN is characterized by extremely severe pain, for which operative intervention is (if temporarily) helpful in most patients.
\end{abstract}

Design: A placebo controlled, parallel, add-on model was developed and the primary outcome variable defined as the length of time during which patients remain in the study. Study groups are compared using Kaplan-Maier survival analysis. Patients record their response to treatment ("severe, moderate, slight, no pain"). The study coordinator monitors pain diaries. Severe or moderate pain of three days duration will result in termination of the study for that patient.

Conclusions: This study design utilizes a method of survival analysis and is novel in chronic pain research. It allows for both early departure from the study and voluntary crossover upon nonresponse. It may be applicable to the analysis of IVIG efficacy in other chronic pain syndromes.

\section{Background}

Patients who are suffering from primary trigeminal neuralgia (pTN) and who have insufficient benefit from medical therapy are frequently referred for operative (microvascular decompression), or neuroablative intervention. Although initial success rates from both methods are high, they are associated with postoperative morbidity [1]. In addition a significant proportion of patients 
experience return of their pain up to several years following these procedures [2].

Patients with TN typically have "sharp, agonizing electricshock-like stabs or pain felt superficially in the skin or buccal mucosa, triggered by light mechanical contact from a more or less restricted site, usually of brief duration - a few seconds (but reportedly occasionally up to 1-2 minutes) and followed by a refractory period of up to a few minutes. Paroxysms may occur at intervals or many times daily or, in rare instances, succeed one another almost continuously "[3]. Pain intensity is mostly extremely severe and is probably among the most intense pains experienced by humans. Therapy with carbamazepine does alleviate symptoms in $60 \%$ of patients [4], but the treatment effect can diminish over time. In addition side effects such as hyponatremia or difficulty with balance may necessitate discontinuation of this medication [2].

We have treated patients with carbamazepine resistant primary trigeminal neuralgia (rpTN) using human pooled intravenous immunoglobulin (IVIG) in a prospective multiple-dose, open-label cohort study [5]. We observed pain free intervals of more than one year duration in five out of ten patients following a median of three treatment cycles [6].

There is clinical evidence, recently reviewed by Latov et al., that IVIG will be effective in some neurological diseases [7]. To date the evidence for a clinically meaningful IVIG efficacy is strongest in Guillain-Barré Syndrome and Chronic Inflammatory Demyelinating Polyneuropathy [8]. However in some cases where there is a purported effect, trials were small and outcomes of dubious relevance. Moreover, methodologically sound trials were often less positive. For instance, though IVIG is considered to be possibly effective in multiple sclerosis, sound studies showed no difference from placebo in reversal of persistent visual loss [9] or established weakness [10]. The benefits we found in an open, uncontrolled study $[5,6]$ might have been skewed by the many biases known to be associated with this study-type and this therefore needs further confirmation using a placebo controlled double blinded trial design.

Challenges for the design of a prospective study include a variable response pattern to IVIG treatment observed in patients [5]. In the preliminary study some patients noted no effect at all, while in patients with a favourable response the pain relief observed was found to last between two weeks and more than one year following a single dose of IVIG. In some patients cumulative benefit was observed with repeated administration of IVIG. Furthermore a 20\% response to placebo has been seen in randomised controlled trials in TN [4].
Here we describe a study-design for a prospective, randomised, double blinded study to evaluate the effect of IVIG in rpTN. The study-hypothesis is that IVIG is more effective than placebo in relieving pain from rpTN. Because of the severity of the pain and the fact that an invasive procedure is known to be effective, participants with no benefit from IVIG would be allowed to leave the study and undergo surgical treatment.

\section{Design \\ Diagnosis and inclusion criteria}

The study has a randomised, double-blind, placebo-controlled, parallel, add-on design in a multicenter, outpatient-based context. Major criteria for a study to be valid are described below. Diagnosis of TN will be made according to International Association for the Study of Pain (IASP) criteria, as described above [3] with one exception: we and others [11] have observed that some patients have additional non dominant, continuous pain in the affected area. Although not specifically mentioned in the IASP criteria, these patients will be included. Patients will be eligible if they have been treated with carbamazepine with insufficient pain relief (see below) or intolerable adverse effects. Unsatisfactory results from treatments with other anticonvulsive drugs [e.g. Lamotrigine, Gabapentin, $[12,13]]$ will be noted. Pre-treatment with these drugs is not essential for study entry. We will include both patients with rpTN who have not yet undergone an invasive procedure (IP) and patients with no more than temporary benefit following an IP. General inclusion/exclusion criteria are listed in Table 1.

\section{Enrolment}

All patients considered candidates for this study will be given a pain diary containing a daily categorical verbal pain intensity scale (VPIS). A pain intensity scale with items severe/moderate/slight/no pain [14] will be used. Each patient will be asked to note in the evening his/her average $24 \mathrm{~h}$ pain intensity using this scale for seven days. In addition the number of bursts of pain (i.e. not individual stabs of pain but paroxysms of pain) and their strength will be noted using an 11-point numeric rating scale. For enrolment pain must be rated severe or moderate everyday, and on at least five days severe. In addition more than 35 pain paroxysms must be documented during this period. If these criteria are fulfilled, this will be judged as insufficient pain relief from established medication. Patients with significant pain relief, but intolerable adverse events with carbamazepine treatment may be included if they have stopped taking carbamazepine, or reduced its intake to a dose where adverse events are tolerable. The entrance criterion of insufficient pain relief must then be fulfilled. 
Table I: General inclusion and exclusion criteria

\begin{tabular}{|c|}
\hline Inclusion criteria \\
\hline Primary idiopathic trigeminal neuralgia \\
\hline Age over 18 \\
\hline Insufficient benefit from carbamazepine therapy \\
\hline Exclusion criteria \\
\hline Serum IgA less than half normal value \\
\hline Pregnancy or breastfeeding mothers \\
\hline Progressive renal failure \\
\hline Bedridden patients \\
\hline Any disorder requiring treatment with IVIG \\
\hline
\end{tabular}

\section{Infection}

We have previously noted an almost incessant return of pain in a large proportion of patients with infection (e.g. flu or cold) who had initially experienced pain relief following IVIG treatment. Therefore, the onset and duration of infection will be noted in all patients and data will later be stratified according to the rate of infection between the groups. Enrolment into the study will be deferred for as long as infection is present.

\section{Blood samples}

The mechanism of action of IVIG has not been elucidated with certainty in any of the disorders, where it currently indicated. Possible mechanisms in TN include the removal of perineural proinflammatory cytokines [15] or the removal of pathological serum antibodies [16]. All patients will be requested to give blood for further analysis before and following IVIG treatment. $40 \mathrm{ml}$ of blood will be taken at the start of the study, 2 weeks following treatment, upon termination/completion of the main study and following the crossover period, if applicable. Serum will be analysed for total IgA (see Table 1) and routine blood chemical values and the remaining will be stored frozen at -20C for later analysis of serum-cytokines and serum antibodies. Peripheral blood cells will be frozen in liquid nitrogen for later cellular studies.

\section{Allocation and blinding}

Patients qualifying for enrolment who give written informed consent after explanation of risks and potential benefits will be allocated a number by the study-coordinator. A computer at a central pharmacy will generate a randomisation schedule and allocation of the active drug or placebo to this number: allocation of patients to treatment schedules will be concealed from investigators. Study drugs for a particular number will be sent on demand directly from the pharmacy to the pharmacy at the peripheral study site. Blinding will be achieved at the pe- ripheral pharmacy by wrapping the infusion bottle in aluminium foil and by wrapping the giving set in an opaque foil. Normal saline will be used as placebo. All patients receive either $30 \mathrm{~g}$ of IVIG, divided into three applications of $10 \mathrm{~g}$ (solubilized in $250 \mathrm{ml}$ sterile water) each and given on alternate days (= one treatment cycle, TC), or placebo (Figure 1). The randomisation code will not be broken until all patients have completed a 28 day study period and a 28 day crossover period, if applicable.

Contact between patients will be discouraged. All patients will receive the following information at enrolment:

1. Both IVIG and placebo may cause an initial pain increase (see below) of up to 4 days duration with no predictive value to the ultimate treatment outcome.

2. Both IVIG and placebo may or may not reduce the pain experienced and both may cause similar adverse events.

3. Pain relief may last as little as a few days, or as long as a month, or even longer in some cases.

4. The study may be left at any time if treatment appears insufficient.

\section{Management of adverse events}

Patients will be informed about possible adverse events upon enrolment. The study coordinator must be contacted should adverse events be observed. As this study will be outpatient based, an observational period at the treatment centre of one hour following the first administration and 30 minutes following consecutive administrations will be required.

\section{Pain and quality of life assessment}

The study period ends 28 days after the first application of IVIG. From the beginning of the study the $24 \mathrm{~h}$ average pain intensity will be noted once daily using the same categorical rating scale as described above. In addition a standard seven point Patient Global Impression of Change Scale (PGIC, [17]) with the following items: much worse/ minimally worse/ no change/ minimally improved/ much improved/ very much improved will be noted along with any adverse events experienced. This impression of change will be referenced to the average status in the week preceding drug administration. Finally the number of paroxysms (see above) and their average strength will be noted using a 24 hourly numeric rating scale diary.

The Medical Outcomes Study 36-Item Short Form (SF36), Dartmouth Primary Care Cooperative Information Project (COOP/WONCA) - Charts for adults, and the Perceived Adjustment to Chronic Illness Scale (PACIS) [18- 


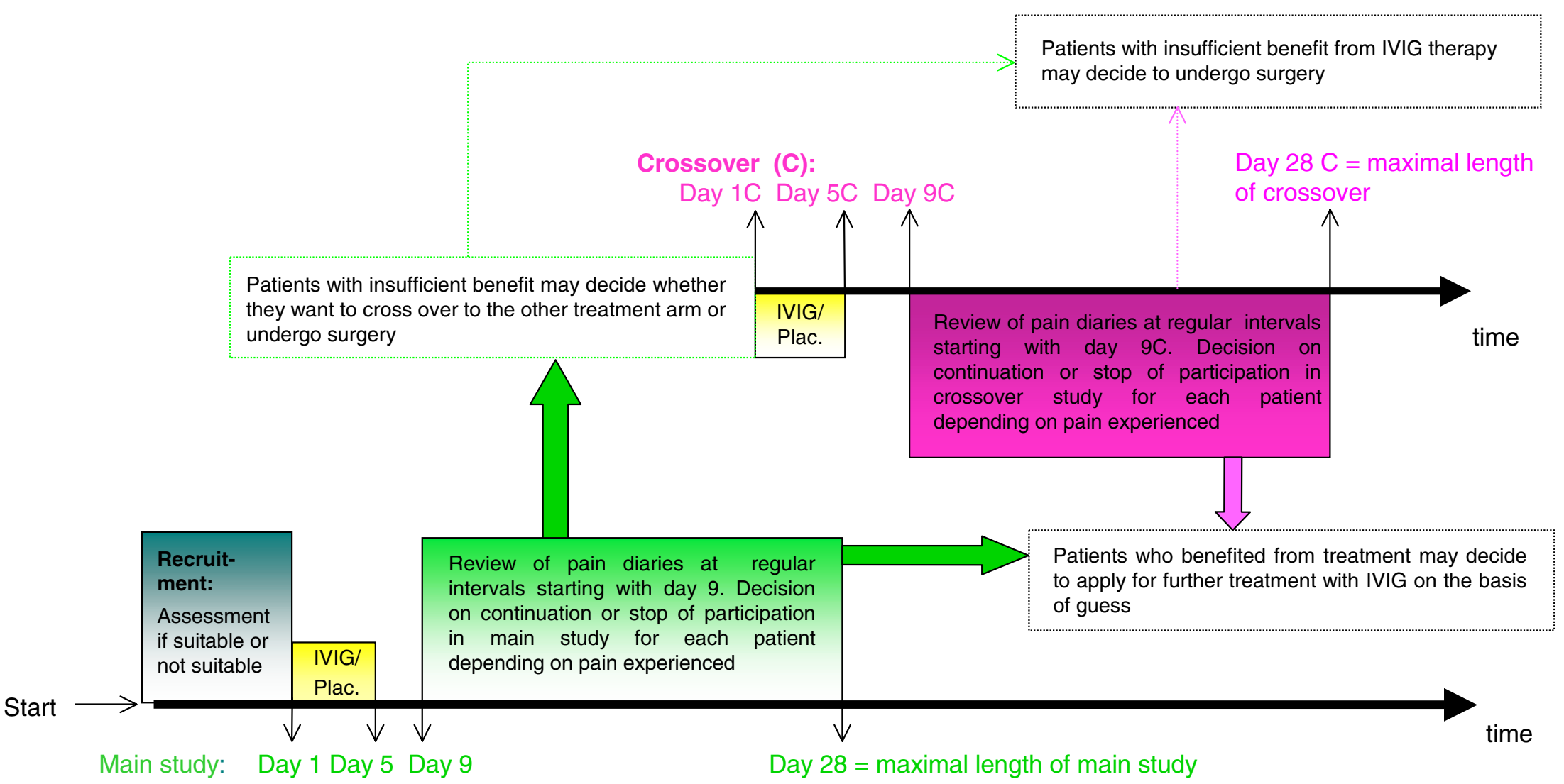

\section{Figure I}

Time flow diagram of the study algorithm. All patients are assessed for 7 days in a recruitment period before commencement of the main study. They receive IVIG or Placebo on alternating days (day I, 3, 5). Subsequently, a three-day period without monitoring is implemented because transitional pain increase has been noted in some patients, with no correlation with the ultimate efficacy of IVIG. Diary entries will be reviewed from day 9 onward. The main study ends at day 28 . Crossover (upper half of diagram): all patients who leave the main study before 28 days will be offered crossover to the alternative treatment arm. Time points (day I C, $3 \mathrm{C}, 5$ $\mathrm{C}$, etc.) are similar to those in the main study. Patients are being offered surgery if they have insufficient pain relief: either upon leaving the main study (as an alternative to crossover), or following crossover treatment. The treatment allocations will be unblinded when all patients have completed the study. Plac: Placebo 
Table 2: Secondary outcome parameters.

\begin{tabular}{|c|c|c|c|c|}
\hline Variable & Groups compared & Scale & $\begin{array}{c}\text { Time period of } \\
\text { evaluation }\end{array}$ & Time compared with \\
\hline Improvement & $A$ & PGIC & $\alpha$ & Week preceding treatment \\
\hline $\begin{array}{l}\text { Reduction in } \\
\text { carbamazepine }\end{array}$ & A & $\begin{array}{l}\text { Fraction of patients with a reduc- } \\
\text { tion of dose; absolute reduction of } \\
\text { dose }\end{array}$ & $\alpha$ & As above \\
\hline Pain & $A$ & I I-point numeric rating scale & $\alpha$ & As above \\
\hline Pain & A & Categorical verbal pain scale & $\alpha$ & As above \\
\hline Pain & A & Average number of attacks & $\alpha$ & As above \\
\hline $\begin{array}{l}\text { Number of patients to } \\
\text { cross over }\end{array}$ & A & $\begin{array}{l}\text { Fraction of patients enrolled in } \\
\text { crossover period }\end{array}$ & At crossover & - \\
\hline Time in study & B & Number of days & Crossover period & Main study period \\
\hline Improvement & B & PGIC & $\alpha$ & $\begin{array}{l}\text { Same time periods post } \\
\text { crossover, as judged against } \\
\text { the week preceding } \\
\text { treatment. }\end{array}$ \\
\hline Pain & B & I I-point numeric rating scale & $\alpha$ & $\begin{array}{l}\text { Same time periods post } \\
\text { crossover }\end{array}$ \\
\hline Pain & B & Categorical verbal pain scale & $\alpha$ & As above \\
\hline Pain & B & Average number of paroxysms & $\alpha$ & As above \\
\hline Quality of life & $A$ & COOP/WONCA charts, SF-36 & $\beta$ & - \\
\hline Quality of life & B & $\begin{array}{l}\text { PACIS, COOP/WONCA charts, } \\
\text { SF-36 }\end{array}$ & $\beta$ & $\begin{array}{l}\text { PACIS: immediately before } \\
\text { first treatment }\end{array}$ \\
\hline
\end{tabular}

A: Patients with placebo and patients with IVIG, B: Patients post crossover and same patients before crossover, $\alpha$ : Average of values from study day 13-19 and average of values during 7 days before termination/ completion of study, $\beta$ : First two weeks following (main or crossover) treatment and last two weeks before the end of (main or crossover) study for a particular patient, PACIS: one time point measurement at day I4 following treatment and at the day of the end of (main or crossover) study. PGIC: Patient Global Impression of Change Scale [17]; SF-36: Medical Outcomes Study 36-Item Short Form [18]; COOP/WONCA: Dartmouth Primary Care Cooperative Information Project charts for adults [I9]; PACIS: Perceived Adjustment to Chronic Illness Scale [20].

20] will be assessed in each patient at the beginning, two weeks after the last IVIG application and upon leaving the study (SF-36: only relevant questions at the last two timepoints, questions will be asked with relation to the "past two", not "past four" weeks).

\section{Concomitant medication}

Doses of pain related drugs given before entry into this study remain unchanged. However, upon discussion with the study coordinator the dosage of pain related drugs may be reduced: it was noted in the above mentioned preliminary study that effective IVIG administration can result in a relative overdosing of concomitant pain related drugs [6]. Reduction of a concomitant carbamazepine dose will be compared between the groups and serves as a secondary outcome parameter (Table 2 ).

\section{Crossover and continuation of treatment}

All patients will be offered crossover to the other treatment upon termination of the main study. For those patients who decide to crossover, same criteria apply as noted above. This "add on study" period ends 28 days following the administration of crossover medication.
Patients who wish continuation of their treatment after the completion of the study because they experience a beneficial effect following treatment and prefer this to an operative procedure will be offered administrative help on the basis of guess [21]. Administrative help will be provided by a health care professional not otherwise involveld in the study, and results of further treatments will be concealed from those health care workers involved in the study until the code is broken. The study coordinator together with the patient will write an application to the appropriate health insurance where the patient is insured. For this it will be assumed that the effective treatment was IVIG. Funding from other sources is ensured in such cases where health insurances decline payment.

\section{Follow up}

All patients will be followed for a period of three years from beginning of the study. They will be asked to note treatments sought for TN during this period and to judge treatment outcome using the above described categorical pain relief scale. Long-term efficacy of IVIG treatment will be researched. In addition a cost analysis will be performed at the end of this period and treatment regiments based on IVIG will be compared with operative and other treatments. 


\section{Primary outcome variable}

The study coordinator will check all pain diaries at regular intervals. This monitoring period will start four days after the administration of the last dose of IVIG (= on the $9^{\text {th }}$ study day). A three-day period without monitoring is implemented because transitional pain increase has been noted in some patients following IVIG administration [5]. In our preliminary, observational study an initial pain increase had no correlation with the ultimate efficacy of IVIG. The primary outcome variable is defined as the length of time patients remain in the study. The length of time in which a patient remains in the study will be decided upon in two ways: the study-coordinator will terminate the study for a patient if, with begin from day 10 the average pain intensity is noted as severe on three consecutive days or if the average pain intensity is noted as moderate and less than "much improved" in the 'impression of change' scale).

In addition, patients can terminate the study at any time. Reasons for termination will be noted. In such cases where patients would not formally withdraw, but undergo an operative procedure or start a new, pain related medication, the time of the new intervention is noted as the time of study termination. Patients, which prematurely terminate the study for other than the above mentioned reasons will be included into an intention to treat analysis.

\section{Secondary outcome variables are listed in Table 2}

Sample size calculation and statistical analysis

All patients and controls (minus dropouts) will remain in the study for at least 11 days (Figure 1). In the remaining 17 study days, it was assumed, that patients treated with the study drug will remain in the study for a median of 10 days from day 12, while patients receiving placebo will remain for a median of 3 days. A sample size calculation was performed, based on the log-rank test. We found $80 \%$ power to detect a hazard value of 3.3 or greater acceptable and assumed a $25 \%$ dropout in both groups. We found that 32 subjects needed to be enrolled in each treatment arm. An intention to treat analysis was agreed to assign dropouts at the study-end. An independent steering committee is scheduled to monitor outcome data at 28 days following treatment of 32 patients. The committee will discontinue the study, if no or less than $20 \%$ median survival advantage is observed in the drug versus placebo group after stratification for dropouts in both groups. At the end of the study, survival data will be analysed using the log-rank test. This will be followed by Cox regression analysis to explore the influence of patient characteristics on IVIG response and to estimate hazard ratios.

\section{Cost}

The cost of this trial has yet to be established in detail.

\section{Discussion}

This study is designed to demonstrate whether the use of IVIG in trigeminal neuralgia is of any clinical benefit. Since most of these patients would normally proceed to have neurosurgical procedures to alleviate their pain when medical management had failed, the choice of primary outcome makes itself. It is whether surgery is avoided over a significant longer period of time in the group treated with the study drug. This choice of outcome has the advantage of being objective rather than subjective, and of being of direct relevance both to patients and to healthcare systems. Avoiding surgery has been used before as the basis of systematic review [22] and economic analysis [23]. There is no alternative other than to perform a randomised trial to test the hypothesis that IVIG is beneficial in trigeminal neuralgia. Firstly, there is no obvious or solid biological mechanism to explain the action of IVIG in trigeminal neuralgia; we begin from the position of perfect equipoise between it working and it not working

An important issue is that even with no effect of IVIG patients should not come to harm. Apart from the fact that they can leave the trial at any time if they feel it does them no good, there are clear clinical stopping rules so that failure of treatment leads to trial of alternative therapy whereby patients initially treated with saline have an opportunity to try IVIG, or they have the option of proceeding to surgery.

Using this novel trial design in chronic pain implies, that a potentially incremental benefit of IVIG with repeated administration [5] cannot be assessed. Follow up data over three years will be vital to gauge the economical equation of IVIG versus surgery. It is important to note that the IVIG dose employed in this trial is a fifth of that in other (autoimmune) diseases and consequently that the cost of treatment is likely to be lower than in these disorders.

\section{Competing interests}

None declared

\section{Authors' contributions}

AG conceived the trial design. He was involved in subsequent adaptations and drafted the manuscript. AM and NR have contributed to adaptations from the original design. AM also participated in drafting the manuscript. RE provided statistical advice and contributed to formulating meaningful outcome parameters. RD and GS contributed to adaptations in the trial design. GS was also involved in choosing an appropriate study group and reviewed the manuscript.

All authors read and approved the final manuscript. 


\section{References}

I. Brisman R Surgical Treatment of Trigeminal Neuralgia. Seminars in Neurology 1997, I 7(4):367-372

2. Barker FG, Jannetta PJ, Bissonette DJ, Larkings MV and Hae Dong Joh The long-term outcome of microvascular decompression for trigeminal neuralgia. The New England Journal of Medicine 1996, 334(17): $1077-1083$

3. Merskey $\mathrm{H}$, Bogduk $\mathrm{N}$ and editors Classification of chronic pain: descriptions of chronic pain syndromes and definitions of pain terms. Seattle IASP Press I994, 59-60

4. McQuay H, Carroll D, Jadad AR, Wiffen P and Moore A Anticonvulsant drugs for management of pain: a systematic review. British Medical Journal 1995, 3 I I: 1047-1052

5. Goebel A, Netal S, Schedel R and Sprotte G Human pooled immunoglobulin in the treatment of chronic pain syndromes. Pain Medicine 2002, 3(2): I 19-127

6. Goebel A, Netal S, Schedel R and Sprotte G Human immunoglobulin (IVIG) treatment of trigeminal neuralgia refractory to carbamazepine-therapy. European Journal of Neuroscience (suppl.) 2000, I 2( I I):360

7. Latov N, Chaudhry V, Koski CL, Lisak RP, Apatoff BR, Hahn AF and Howard JF Jr Use of intravenous gammaglobulins in neuroimmunologic diseases. The Journal of Allergy and Clinical Immunology 200I, I08(4 suppl): I26-32

8. Dalakas MC Intravenous Immunoglobulin in the treatment of autoimmune neuromuscular diseases: present status and practical therapeutic guidelines. Muscle-Nerve 1999, 22:14791497

9. Noseworthy JH, O'Brien PC, Petterson TM, Weis J, Stevens L, Peterson WK, Sneve D, Cross SA, Leavitt JA and Auger RG A randomised trial of intravenous immunoglobulin in inflammatory demyelinating optic neuritis. Neurology 200I, 56(I I): I5 I4-1522

10. Noseworthy JH, O'Brien PC, Weinshenker BG, Weis JA, Petterson TM, Erickson BJ, Windebank AJ, Whisnant JP, Stop-Smith KA and Harper CM IV immunoglobulin does not reverse established weakness in MS. Neurology 2000, 55(8): I | 35- I | 43

II. Nurmikko T] and Eldridge PR Trigeminal neuralgia - pathophysiology, diagnosis and current treatment. British Journal of Anaesthesia 2001, 87:117-132

12. Zakrzewska JM, Chaudhry Z, Nurmikko TJ, Patton DW and Mullens EL Lamotrigine (Lamictal) in refractory trigeminal neuralgia: results from a double - blind placebo controlled crossover trial. Pain 1997, 73:223-230

13. Sindrup $\mathrm{SH}$ and Jensen TS Pharmacotherapy of trigeminal neuralgia. Clinical Journal of Pain 2002, I 8(I):22-27

14. McQuay $\mathrm{H}$ and Moore A An evidence-based resource for pain relief. Oxford University Press, Oxford, New York 2000, I4:

15. Sommer C, Schafers M, Marziniak M and Toyka KV Etanercept reduces hyperalgesia in experimental painful neuropathy. Journal of the Peripheral Nervous System 200I, 6(2):67-72

16. Goebel A, Steinbach H, Karch H, Schedel R, Arnold A, Roewer N and Sprotte $G$ (Minimal seroconversion rates following antibiotic treatment of patients with chronic pain syndromes and IgA seroantibodies to Campylobacter jejuni or human pathogenic Yersiniae) (German). Der Schmerz 200I, I 5(I):S7I-S72

17. Farrar JT, Young JP, LaMoreaux L, Werth JL and Poole RM Clinical importance of changes in chronic pain intensity measured on an I I-point numerical pain rating scale. Pain 200I, 94: I49-I 58

18. Ware JE, Snow KK Jr, Kosinski M and Gandek B SF-36 Health Survey Manual and Interpretation Guide. New England Medical Center, Boston, MA 1993,

19. Nelson EC, Landgraf JM, Hays RD, Wasson JH and Kirk JW The functional status of patients: how can it be measured in physicians' offices? Medical Care 1990, 28: I I I I-I I26

20. Hürny $C h$, Jürg $B$, Bacchi $M$, van Wegberg $B$, Tomamichel $M$, Spek $U$, Coates A, Castiglione M, Goldhirsch A and Senn HJ The perceived adjustment to chronic illness scale (PACIS): a global indicator of coping for operable breast cancer patients in clinical trials. Supportive Care in Cancer 1993, I:200-208

21. Dalakas MC, Illa I, Dambrosia JM, Soueidan SA, Stein DP, Otero C, Dinsmore ST and McCrosky S A controlled trial of high-dose intravenous immune globulin infusions as treatment for dermatomyositis. The New England Journal of Medicine 1993, 329(27): 1993-2000
22. Moore RA, Commins DJ, Bates G and Phillips CJ S-carboxymethylcysteine in the treatment of glue ear: quantitative systematic review. BMC Family Practice 200I, 2:3

23. Phillips CJ, Burrell A, Moore RA, Bates GJ and Commins D Mucodyne: the economics of preventing surgery for grommets. Journal of Medical Economics 1999, 2:167-176

\section{Pre-publication history}

The pre-publication history for this paper can be accessed here:

http://www.biomedcentral.com/1471-2377/3/1/prepub
Publish with Bio Med Central and every scientist can read your work free of charge

"BioMed Central will be the most significant development for disseminating the results of biomedical research in our lifetime. "

Sir Paul Nurse, Cancer Research UK

Your research papers will be:

- available free of charge to the entire biomedical community

- peer reviewed and published immediately upon acceptance

- cited in PubMed and archived on PubMed Central

- yours - you keep the copyright

Submit your manuscript here:

http://www.biomedcentral.com/info/publishing_adv.asp
BioMedcentral 